\title{
PVP2015-45345
}

\section{PROBABILISTIC ASSESSMENT OF INNOVATIVE MITIGATING MEASURES FOR BURIED STEEL PIPELINE - FAULT CROSSING}

\author{
Vasileios E. Melissianos \\ JB ATE \\ 30, Itanou Str. \\ GR-71307, Herakleion, Crete, Greece \\ Email: melissianosv@gmail.com
}

\author{
Dimitrios Vamvatsikos \\ National Technical University of Athens \\ School of Civil Engineering \\ 9, Iroon Polytechneiou Str., Zografou Campus \\ GR-15780, Athens, Greece \\ Email:divamva@mail.ntua.gr
}

\author{
Charis J. Gantes \\ National Technical University of Athens \\ School of Civil Engineering \\ 9, Iroon Polytechneiou Str., Zografou Campus \\ GR-15780, Athens, Greece \\ Email: chgantes@central.ntua.gr
}

\section{ABSTRACT}

A methodology is presented on assessing the effectiveness of flexible joints in mitigating the consequences of faulting on buried steel pipelines through a comprehensive analysis that incorporates the uncertainty of fault displacement magnitude and the response of the pipeline itself. The proposed methodology is a two-step process. In the first step the probabilistic nature of the fault displacement magnitude is evaluated by applying the Probabilistic Fault Displacement Hazard Analysis, considering also all pertinent uncertainties. The second step is the "transition" from seismological data to the pipeline structural response through the fault displacement components as the adopted vector intensity measure. To mitigate the consequences of faulting on pipelines, flexible joints between pipeline parts are proposed as innovative measure for reducing the deformation of pipeline walls. Thus, the mechanical behavior of continuous pipelines and pipelines with flexible joints is numerically assessed and strains are extracted in order to develop the corresponding strain hazard curves. The latter are a useful engineering tool for pipeline fault crossing risk assessment and for the effectiveness evaluation of flexible joints as innovative mitigating measures against the consequences of faulting on pipelines.

\section{INTRODUCTION}

Onshore buried steel pipelines extend to long distances, often crossing seismic areas that incorporate active faults. Thus, the potential for imposed large ground displacements on the pipeline has to be considered in the design, since past earthquake events have demonstrated that the dominant cause of pipeline seismic damage is fault offset [1]. The principal failure modes in such cases are local buckling of the pipeline wall due to compressive strains and tensile fracture of girth welds between adjacent pipeline parts due to tensile strains. Acknowledging that pipelines are hazardous structures whose potential failure may heavily affect the environment, nearby populated areas and the economy, it is deemed appropriate on one hand to perform a comprehensive risk analysis of the pipeline - fault crossing and on the other hand to propose effective mitigating measures in order to relieve the consequences of faulting on the pipeline.

However, fault displacement is a naturally random event that raises the question as to what is the appropriate magnitude of ground displacement that has to be considered in the design of a pipeline. Two approaches are available at this point. The first is the deterministic one, where a particular fault displacement "worst case" scenario is considered, consisting of a postulated occurrence of an earthquake with a specific magnitude at a specific location, without providing further information regarding the expected level of fault offset during the pipeline life-cycle. Typically, the effects of uncertainties encountered in the various design steps are neglected. Recognizing, then, that our knowledge on the fault movement recurrence is incomplete, the adoption of the second approach, the so called probabilistic one, is necessary. 
Among the two approaches, the latter is herein adopted in order to achieve a balance between safety and economy. So, the proposed procedure for risk assessment of innovative measures consists of two parts: (i) conduct the probabilistic analysis of fault displacement hazard, (ii) perform pipeline structural analysis and then merge the results to conduct the seismic risk analysis. Some aspects of the theoretical background of the proposed procedure for pipeline - fault crossing have been presented in previous work of the authors [2]. Then, regarding the first task, the appropriate tool is the Probabilistic Fault Displacement Hazard Analysis (PFDHA) introduced by Youngs et al. [3], which aims at quantifying the mean annual rate of exceeding various fault displacement levels at the site of pipeline - fault crossing. PFDHA integrates available geological and seismological data including, for example, the probability of fault activation, rupture location, fault slip rate, distribution of earthquake magnitudes and their associated uncertainties. Then, combination of results through seismic risk analysis leads to the development of hazard curves for the selected intensity measure and the efficiency evaluation of different pipeline designs or characteristics.

Buried pipeline response to faulting is related to the fault type, the fault dip angle and the pipeline - fault intersection angle. It is generally accepted that slender steel members perform better in tension that in compression, as the latter is associated with buckling susceptibility. However, large fault offsets lead to pipe bending, shear and tension/compression, thus the combined effect of actions has to be evaluated in order to assess the pipeline mechanical behavior. For that purpose the pipeline design against fault movement is commonly carried out in strain rather than stress terms.

Mitigating the consequences of faulting on buried steel pipelines remains a top research topic for both the academia and the industry. Common design approaches nowadays include, among others, embedment of the pipeline around the fault zone in a shallow trench with loose backfill [4] and the use of geotextiles, which are wrapped around the pipe [5]. These measures aim at reducing pipe - soil friction, allowing the development of high strains, provided that rupture is prevented and the internal pressure boundary is maintained. However, the effectiveness of these approaches has not been fully quantified [6]. Recently, research is directed towards integrating expansion joints between adjacent pipe parts in the vicinity of fault crossing, in order to concentrate strains at the joints and retain the steel pipe nearly unstressed [7], [8]. This design approach is completely different from the commonly used friction reduction approaches. Flexible joints are part of the structural system and in fact transform the continuous pipeline to a segmented one, while at the same time there is no need for special requirements regarding trench construction and pipe wrapping.

\section{FAULT DISPLACEMENT HAZARD ANALYSIS}

The geometry of pipeline - fault crossing is depicted in Fig. 1 both in section and plan view. The global coordinate system $(1,2,3)$ refers to spatial fault movement, while $(\mathrm{x}, \mathrm{y}, \mathrm{z})$ is the local coordinate system of the pipe. Then, $\beta$ and $\psi$ are the pipeline - fault crossing angle and fault dip angle, respectively, $L F$ is the fault length and $L_{p}$ is the crossing site distance from the closest fault edge. The magnitude of fault movement is denoted by $D$ and its spatial components are defined by $\Delta_{1}, \Delta_{2}$ and $\Delta_{3}$. Also, the magnitude of the imposed displacements on the pipeline, namely $\Delta_{x}, \Delta_{y}$ and $\Delta_{z}$, are obtained, through the rotation of the global coordinate system by the angle $\beta$ :

$$
\begin{aligned}
& D=\sqrt{\Delta_{1}^{2}+\Delta_{2}^{2}+\Delta_{3}^{2}}, \text { with } \Delta_{3}=\Delta_{2} \tan \psi \\
& \Delta_{x}=\Delta_{1} \cos \beta+\frac{\Delta_{3}}{\tan \psi} \sin \beta \\
& \Delta_{y}=-\Delta_{1} \sin \beta+\frac{\Delta_{3}}{\tan \psi} \cos \beta \quad \Delta_{z}=\Delta_{3}
\end{aligned}
$$

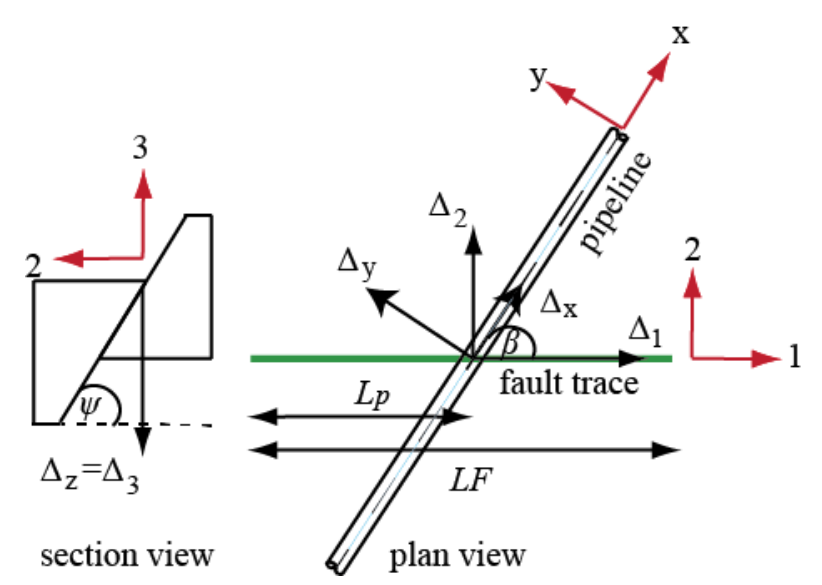

Fig. 1 Pipe - fault crossing section and plan view

Youngs et al. [3] introduced the basis of PFDHA and proposed two discrete approaches within it, the "earthquake approach" and the "displacement approach". The former is derived from Probabilistic Seismic Hazard Analysis [9] and relates the occurrence of fault displacement for a site at or near the ground surface to the occurrence of earthquakes in the site region. The "displacement approach" needs extensive recorded data or paleoseismic data that is typically absent for most sites. Thus, the first approach is adopted. Moreover, only principal faulting is considered, without taking into account distributed faulting issues [3].

In terms of practical applications, fault displacement hazard estimation depends on three factors: (i) earthquake magnitude, (ii) length of rupture and (iii) rupture position along the fault trace. Among them, earthquake magnitude stands as the key factor for describing a seismic source, which ranges from a minimum value $\left(M_{\min }\right)$ of engineering significance to a maximum value $\left(M_{\max }\right)$ as constrained by the finite fault size, while the range of values is discretized into a number of bins to account for all possible values. Acknowledging, then, that 
different earthquakes may rupture fault lengths of different size, the surface rupture length (SRL) along the fault trace is introduced as the second factor. Then, as the location of a given earthquake determines whether the rupture crosses the pipeline site, the third factor regarding SRL position on the fault trace is considered. Thus, a variety of potential SRLs is taken into account, each at a different location. Without more detailed data, SRLs of the same size are considered to be equiprobable. For simplicity with bookkeeping, a minimum SRL size is determined, e.g., as corresponding to the minimum magnitude of interest via empirical equations [10], and all subsequent larger sizes are simply integer multiples. So, practically every SRL size is accounted for at all possible positions, keeping track of those where the rupture intercepts the pipeline and contributes to the pipeline displacement hazard.

PFDHA is implemented in the present study as an application of the total probability theorem. The mean annual rate of exceedance of a given fault displacement value $d$ at a pipeline crossing site is expressed as $\lambda_{D}(d)$. In general, by $\lambda_{X}(x)$ we mean the annual rate of variable $X$ exceeding value $x$. Therefore, $\lambda_{D}(d)$ is a summation over all possible distinct scenarios that could produce an exceedance, using earthquake magnitude $M$ as the conditioning variable:

$$
\lambda_{D}(d)=v_{o} \sum_{i} P\left(D>d \mid m_{i}\right) \lambda_{M}\left(m_{i}\right)
$$

$v_{o}$ stands for the rate of all earthquakes above $M_{\min }$ and $\lambda_{M}\left(m_{\mathrm{i}}\right)$ is the magnitude occurrence frequency according to the Gutenberg-Richter Bounded Recurrence Law [11]. The probability function $P\left(D>d \mid m_{\mathrm{i}}\right)$ estimates the probability that fault displacement exceeds a defined value $d$ given earthquake magnitude $m_{i}$ and is summed over all magnitude values:

$$
\begin{aligned}
P\left(D>d \mid m_{i}\right)= & \\
& \sum_{i} \sum_{j} \sum_{k} P\left(D>d \mid m_{i}, S R L_{j}, F D_{k}, P o s_{l}\right) \times \\
& P\left(S R L_{j}, F D_{k} \mid m_{i}\right) \times 1 / N_{l}
\end{aligned}
$$

In Eq. (4), apart from earthquake magnitude range discretization in $i$ bins and rupture length discretization in $j$ bins, a third discretization for the fault displacement (FD) option of PFDHA is incorporated. It is noted that the proposed algorithm is applied similarly for the average $(A D)$ or the maximum $(M D)$ displacement approach. The right side of Eq. (4) consists of three terms. The first one is the conditional probability of exceedance $P\left(D>d \mid m_{i}, S R L_{j}, F D_{k}, P o s_{l}\right)$, which stands as the core of the PFDHA and necessitates detailed calculations that are carried out over each combination of bins of earthquake magnitude, rupture length, fault displacement and all possible positions of $S R L_{j}$ along the fault trace. The last term is the probability of each SRL location, considered to be an independent variable for a given magnitude. Positions of samesize $S R L_{j}$ are assumed equiprobable, corresponding to a probability $1 / N_{l}$, with $N_{l}$ being the total number of $S R L_{j}$ positions of a given length. Finally, the middle term is the probability of a given range of fault displacement and size of SRL to occur given the magnitude. $S R L_{j}$ and $F D_{k}$ are well correlated given the earthquake magnitude. So, $P\left(S R L_{j}, F D_{k}, \mid m_{i}\right)$ $=P_{i, j, k}$ is estimated using the well-known joint distribution provided by Wells and Coppersmith [10] that allows the definition of the corresponding probability density function (PDF) of $f\left(S R L_{j}, F D_{k} \mid m_{i}\right)$ as a joint lognormal with positive correlation. Assuming that the discretization is sufficiently fine, the probability of $S R L$ and $F D$ falling in a bin given the magnitude can be approximated via a single PDF value at its center:

$$
\begin{aligned}
P_{i, j, k} & =\int_{F D_{k}-\frac{F D_{\text {step }}}{2}}^{F R L_{j}-\frac{F D_{\text {step }}}{2} S R L_{j}+\frac{S R L_{\text {step }}}{2}} \int_{\text {sep }}^{2} f\left(S R L_{j}, F D_{k} \mid m_{i}\right) d F D d S R L \\
\cong & f\left(S R L_{j}, F D_{k} \mid m_{i}\right) \Delta F D \Delta S R L
\end{aligned}
$$

Still, the sum of $P_{i, j, k}$ over $j$ and $k$ for all $S R L_{j}$ and $F D_{k}$ should equal one. Thus, the derived probability mass function approximation is appropriately renormalized:

$$
P\left(S R L_{j}, F D_{k} \mid m_{i}\right)=P_{i, j, k} / \sum_{j} \sum_{k} P_{i, j, k}
$$

\section{FAULT DISPLACEMENT COMPONENTS}

In seismic risk analysis the roles of the seismologist and the structural engineer are linked by the use of an interface variable, known as the intensity measure (IM). For pipeline - fault crossing assessment the structural engineer needs to estimate the response of the structure to given IM values. The proper such values are the fault displacement spatial components $\Delta_{1}, \Delta_{2}$ and $\Delta_{3}$. Thus, an appropriate vector IM [12] need only consists of two components $\left[\Delta_{1}, \Delta_{3}\right]$, as the third one is functionally dependent on them. Additionally, as the fault is assumed to be planar, fault displacement components $\Delta_{1}$ and $\Delta_{3}$ are structurally independent variables and are also adequate to fully describe the structural model loading condition.

However, there is no sufficient data and information regarding their distribution with reference to the fault displacement norm. Thus, a simple and approximate calculation procedure is proposed. Thereafter, whether the fault type is normal/reverse or strike-slip, the independent fault component $\Delta_{3}$ or $\Delta_{1}$, respectively, is assessed assuming a uniform distribution ranging from $0.7 D$ to $0.9 D$. The range limits adopted are based on fault characterization and the fact that fault movement in nature tends to be three dimensional, given that the fault type is simply characterized by the dominant component. So, the introduction of a fault displacement component distribution enables the estimation of fault displacement vector IM. Thus, sampling of equiprobable triplets of individual components $\left[\Delta_{1}, \Delta_{2}, \Delta_{3}\right]$ is carried out. 
Then, assuming, for example, a strike-slip fault, the Mean Annual Rate of Density for fault components is estimated as:

$$
\lambda^{\prime}\left(\Delta_{1}, \Delta_{3}\right)=f\left(\Delta_{1} \mid D\right) \times \frac{d \lambda_{D}}{d D} \times \frac{\partial \Delta_{3}}{\partial D}
$$

where $f\left(\Delta_{1} \mid D\right)$ is the PDF of the distribution of $\Delta_{1}$ values given the fault displacement norm $D, \mathrm{~d} \lambda_{D} / \mathrm{d} D$ is the rate density for given $D$, while $\partial \Delta_{3} / \partial D$ is the derivative of $\Delta_{3}$ over $D$ that is necessary to change the variable. It should be noted that $\lambda^{\prime}\left(\Delta_{1}, \Delta_{3}\right)$ is not rate, but rate density and an integration of $\lambda\left(\Delta_{1}, \Delta_{3}\right)$ over a two-dimensional interval of $\Delta_{1}$ and $\Delta_{3}$ will result to the mean annual rate of events occurring in this interval.

\section{UNCERTAINTY ANALYSIS}

The probabilistic nature of the problem necessitates the consideration of uncertainties in the seismic hazard analysis, since the use of the mean hazard curve is highly sensitive to extreme scenarios [13]. From its origins, PFDHA can incorporate any quantifiable uncertainty, and thus in the present study uncertainties related to the inadequate understanding of the nature of seismological parameters are considered. In practice, uncertainties lead to alternative hazard curves and are handled through logic trees, where weight factors are assigned to the different branches. These factors are selected in such a way that they are not frequency-based probabilities, acknowledging that tree branches represent the engineer's degree of belief in the alternative models [14].

In the present study, the logic tree of Fig. 2 is adopted, to account for seismic rate $v$, maximum earthquake $M_{\max }$ and displacement option of PFDHA, either maximum (MD) or average (AD) [3]. Seismic rate is the dominant feature of the seismic source and is related to high uncertainty, as its mean value is usually provided by seismologists. Maximum earthquake magnitude is also provided by seismologists and is under question, unlike minimum magnitude that is chosen by decision, assuming that lower values do not contribute to seismic hazard. Finally, within the PFDHA the use of either the average or the maximum fault displacement for normalizing fault displacement data sets throughout calculation of the ground motion prediction equation is a viable approach.

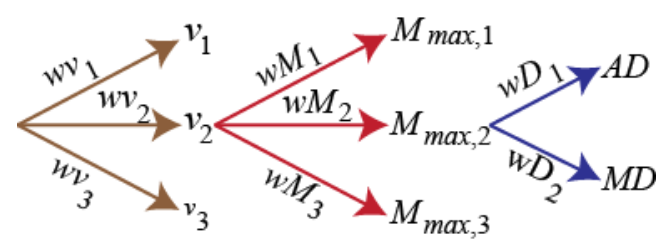

Fig. 2 Uncertainty analysis logic tree

\section{PIPELINE STRUCTURAL ANALYSIS}

Analysis and design of buried pipelines against fault offset is directly related to soil nonlinear behavior, while pipe - soil interaction increases further the complexity of the problem. Thus, the implementation of advanced numerical modeling techniques is inevitable. There are two pertinent numerical simulation techniques: (i) the beam-type FEM model, where the pipeline is modeled with beam-type finite elements and the surrounding soil with nonlinear translational springs, and (ii) the continuum models, where the pipeline is meshed with shell elements and the surrounding soil with solid three-dimensional elements. The continuum model is considered to be a more advanced approach, as local buckling of the cylinder wall can be assessed, more accurate soil models can be implemented and trench boundaries can be incorporated in the analysis [15], [16], [17]. However, this modeling technique increases drastically the complexity of the model, the computational effort and the problem nonlinearity, given the need for contact elements in modeling pipeline - soil interaction, which can cause convergence problems. So, its applicability in practice is limited. Thus, in the present study the generally accepted beamtype finite element model is adopted based on its acceptable accuracy, reliability and minimum computational effort, as it provides the ability to capture, directly or indirectly, the main effects and failure mechanisms. So, the pipeline is modeled with beam-type elements that can simulate its bending and axial deformation and can also provide stresses and strains on crosssections along its axis. Then, soil is represented by a series of mutually independent uniaxial nonlinear springs in three directions, as illustrated in Fig. 3. Axial springs simulate the pipe - soil friction and their properties depend on backfill properties and the pipeline coating material. Lateral horizontal springs simulate soil resistance to any pipeline transverse movement in the trench, while upward and downward springs model the soil resistance to vertical pipeline movement, with their properties differing significantly.

Flexible joints are proposed as innovative mitigating measures against pipe damage due to fault movement. They are commercial products, usually referred as bellows, and are used in the piping industry to absorb thermal expansion and thrust. The two main bellow types are the single joint with axial, lateral and angular deformation capability and the hinged joint with angular deformation only. For buried pipeline applications hinged-type joints allowing rotations but restricting axial and transverse displacements are selected, as axial and lateral stiffness of single-type commercial joints are not high enough to withstand large fault displacements. Hinged bellows can be modeled either as a generic flexible joint represented by a rotational spring at its center point, without modeling its length, or as a general beam finite element with a stiffness matrix derived from the spring constants provided by the manufacturer [18]. In this study, the first simulation approach is adopted by modeling hinged bellows as an elastic rotational spring with stiffness $0.0088 \mathrm{kNm} / \mathrm{deg}$, while relative axial, lateral and torsional movements at the two ends of the bellow are restrained through constraint equations. Finally, the locations of flexible joints are selected based on the location of maximum bending moment of the continuous pipeline due to fault 
activation. Three flexible joints are integrated into the pipeline, one on the fault, one in the footwall part and the third in the hanging wall part of the fault.

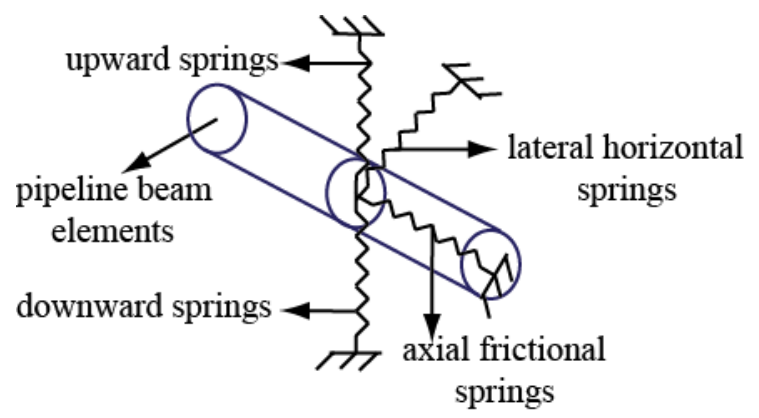

Fig. 3 Beam-type finite element model

\section{STRAIN HAZARD CURVES}

Fault activation causes differential ground movement that imposes large permanent ground displacement on buried pipelines. Such displacements heavily endanger the pipeline's integrity and may lead to fracture and leakage. Hence, pipe design aims at controlling strain demands against strain capacities. Strain hazard curves are the appropriate tool for performing a probabilistic estimation of any potential failure. While numerical analyses provide strain demands, strain capacity terms are adopted based on code recommendations. ALA [19] provisions suggest the tensile limit $\varepsilon_{t, c}=2 \%$ and the compressive limit $\varepsilon_{c, c}$ for longitudinal strains resulting from ground movement due to fault offset:

$$
\begin{gathered}
\varepsilon_{c, c}=0.5\left(\frac{t}{D^{\prime}}\right)-0.0025+3000\left(\frac{p D_{e}}{2 E t}\right)^{2} \\
\text { where } D^{\prime}=D_{e} /\left[1-3\left(D_{e}-D_{\min }\right) / D_{e}\right]
\end{gathered}
$$

$t$ is the pipeline wall thickness, $D_{e}$ is the pipeline external diameter, $D_{\min }$ is the internal diameter, $p$ is the internal pressure and $E$ the pipeline steel modulus of elasticity. Eq. (8) includes a term for internal pressure, which acts as a relief against the external earth pressure. In the present study the internal pressure is assumed to be equal to zero, as a less favorable situation, thus the pertinent term is neglected. Strain hazard curves present the mean annual rate of exceedance of a defined strain value and are evaluated as:

$$
\lambda_{E}(\varepsilon)=\sum_{\varepsilon\left(\delta_{1 i}, \delta_{3 j}\right) \geq \varepsilon} \Delta \lambda\left(\delta_{1 i}, \delta_{3 j}\right)
$$

where $\Delta \lambda\left(\delta_{1 i}, \delta_{3 j}\right)$ is the mean annual rate of equaling (or reaching) a given range of fault displacement components $\delta_{1 i}$ and $\delta_{3 j}$. This is estimated by integrating the $\lambda^{\prime}\left(\Delta_{1}, \Delta_{3}\right)$ of Eq. (7) in the appropriate range of displacements $\delta_{1 i}$ and $\delta_{3 j}$. Finally, this result is in turn summed over all $i$ values of $\Delta_{1}$ and $j$ values of $\Delta_{3}$ that are found via structural analysis to correspond to strain values larger than $\varepsilon$.

\section{ILLUSTRATIVE EXAMPLE}

To illustrate the proposed methodology, a numerical example is presented. For that purpose a strike-slip fault is considered with length $L F=100 \mathrm{~km}$, fault dip angle $\psi=70^{\circ}$, and a pipeline crossing site at a distance of $L_{p}=40 \mathrm{~km}$. The pipeline - fault crossing angle is $\beta=80^{\circ}$, referring to the notation of Fig. 1, while the seismological and uncertainty parameters of the model are summarized in Table 1. The fault offset is assumed to take place in a horizontal plane, thus fault displacement $D$ is equal to $\Delta_{1}$, while the other two components are $\Delta_{3}=\Delta_{2}=0$. Fault displacement values under examination range from $0.1 \mathrm{~m}$ up to $3.0 \mathrm{~m}$. Additionally, as the pipeline intercepts the fault with angle $\beta=80^{\circ}$, pipeline displacement components $\Delta_{x}$ and $\Delta_{y}$ are extracted from Eq. (2) and are univocally related to $\Delta_{1}$, while $\Delta_{z}=0$.

\section{Table 1 Seismological and uncertainty parameters}

\begin{tabular}{|ll|ll|}
\hline$M_{\max , 1}=7.2$ & $w M_{1}=0.2$ & $v_{1}=1.0$ & $w v_{1}=0.3$ \\
$M_{\max , 2}=7.3$ & $w M_{2}=0.6$ & $v_{2}=1.2$ & $w v_{2}=0.4$ \\
$M_{\max , 3}=7.4$ & $w M_{3}=0.2$ & $v_{1}=1.4$ & $w v_{3}=0.3$ \\
\hline$M_{\min }=5.0$ & & $A D$ & $w D_{1}=0.5$ \\
& & & \\
\cline { 3 - 4 } & &
\end{tabular}

Implementing PFDHA through the previously described algorithm provides the $\lambda_{D}$ of fault offset values on pipeline crossing, as shown in Fig. 4, with the descending curve shape being predictable, as the larger the fault displacement is, the lower the $\lambda_{D}$. However, given that the fault offset is limited to the $\Delta_{1}$ fault component, which equals $D$, for our purposes, Eq. (9) yields $\lambda_{\mathrm{E}}(\varepsilon)=\lambda(D(\varepsilon))$, where $D(\varepsilon)$ is the function that relates the fault displacement $(D)$ with the strain $\varepsilon$ obtained from pipeline structural analysis.

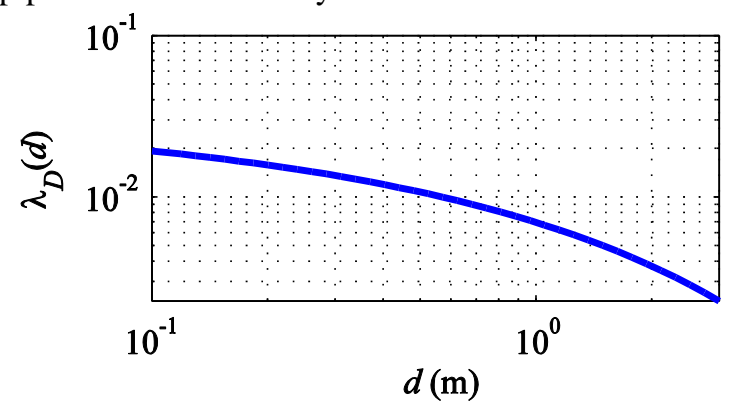

Fig. 4 Mean annual rate of exceeding fault displacement on pipeline crossing site

Pipeline numerical simulation is carried out using the commercial FEM software ADINA [20]. A typical high-pressure large-diameter transmission pipeline is considered, featuring a cross-section with external diameter $D_{e}=914 \mathrm{~mm}$, wall thickness $t=12.7 \mathrm{~mm}$ and a total modeled length of $L=1000 \mathrm{~m}$, while the fault is assumed to intercept the pipeline in its middle- 
span. Steel is of type API5L-X65 and considered as elastic plastic with isotropic hardening with yield stress $f_{y}=448.5 \mathrm{MPa}$, ultimate stress $f_{u}=531.0 \mathrm{MPa}$, elastic modulus $E=210 \mathrm{GPa}$ and ultimate strain $\varepsilon_{u}=4.0 \%$. The pipeline is considered to be coated with coal tar and embedded under $1.3 \mathrm{~m}$ of loose sand with cohesion $c=0$, unit weight $\gamma=18 \mathrm{kN} / \mathrm{m}^{3}$ and internal friction angle $\varphi=36^{\circ}$. Regarding numerical modeling, the pipeline is meshed with PIPE elements of length $0.5 \mathrm{~m}$, after a mesh density sensitivity analysis was carried out. The surrounding soil is modeled with elastic - perfectly plastic spring elements whose properties are estimated according to ALA [19] provisions. The differential ground movement is applied statically on the fault hanging wall, as imposed displacement on the corresponding "ground" nodes of spring elements. "Ground" nodes of spring elements on fault footwall are considered fixed. Then, as fault offset may be in the order of meters, geometrical nonlinearity of the problem is taken into account to consider second order effects, as well as materially nonlinearity to account for steel and soil nonlinear behavior.

As an example to illustrate the effectiveness of flexible joints, numerical results from a single analysis of a continuous pipeline (abbreviated as $\mathrm{CP}$ ) and a pipeline with flexible joints (abbreviated as PFJ) are presented, where the pipeline is subjected to $1.9 \mathrm{~m}$ of fault displacement. The deformations of $\mathrm{CP}$ and PFJ due to strike-slip faulting are illustrated in Fig. 5, indicating that $\mathrm{CP}$ deformation is a smooth curved line, while PFJ deformation consists of straight line segments around the fault, given that flexible joints act almost as internal hinges.

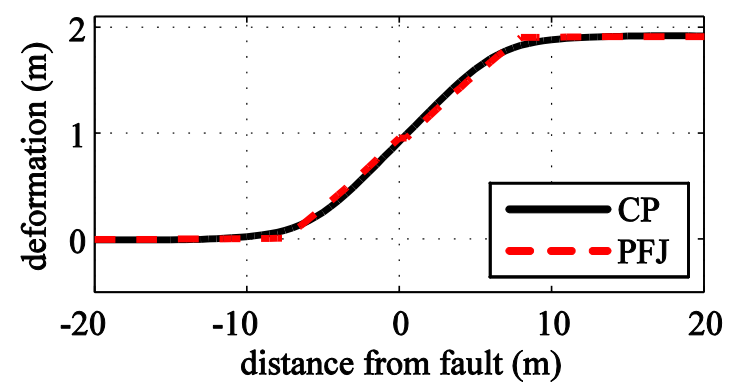

Fig. 5 Deformation of continuous pipeline and pipeline with flexible joints

The bending moment distributions along the pipeline (Fig. 6) demonstrate a major decrease in moment, while the axial force distributions (Fig. 7) indicate a minor increase, as a balance has to be achieved with reference to structural global stiffness. The distribution of maximum longitudinal strains along the pipeline (Fig. 8) demonstrate the significant decrease of strains due to joints, as strains are concentrated at the joints, while much lower strains develop on pipe steel parts, thus reducing the risk of compressive or tensile failure, which is the design objective.

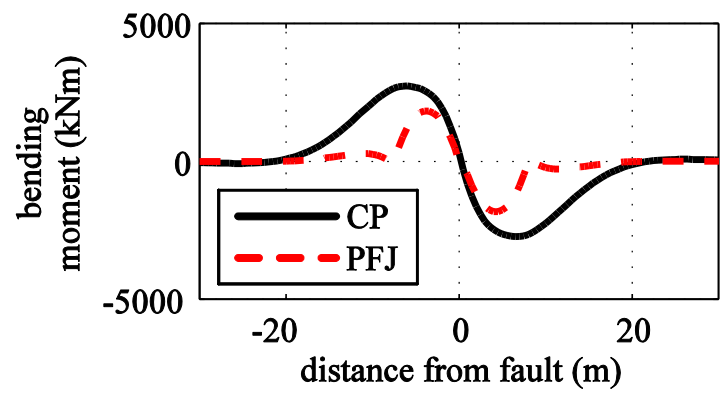

Fig. 6 Bending moment distribution of continuous pipeline and pipeline with flexible joints

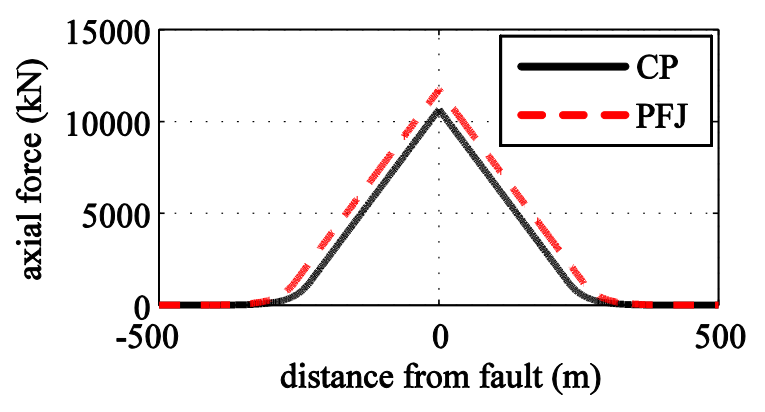

Fig. 7 Axial force distribution of continuous pipeline and pipeline with flexible joints

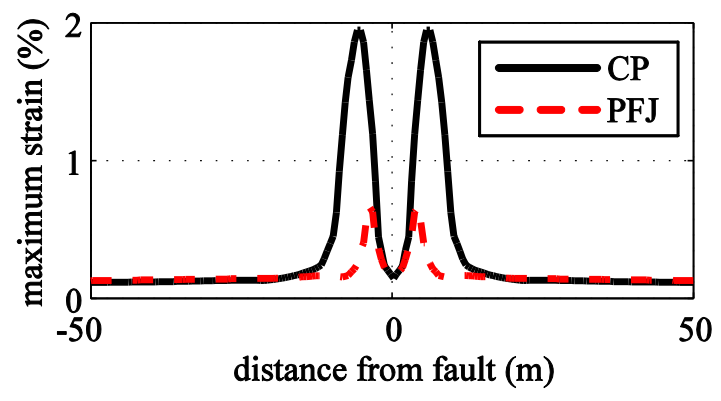

Fig. 8 Longitudinal strain distribution of continuous pipeline and pipeline with flexible joints

With reference to soil response due to pipeline movement, Fig. 9 illustrates lateral soil response distribution along $\mathrm{CP}$ and PFJ. The integration of flexible joints does not modify significantly the length of soil plastification, as soil force development is due to pipeline relative movement in the trench, which is similar in both cases. 


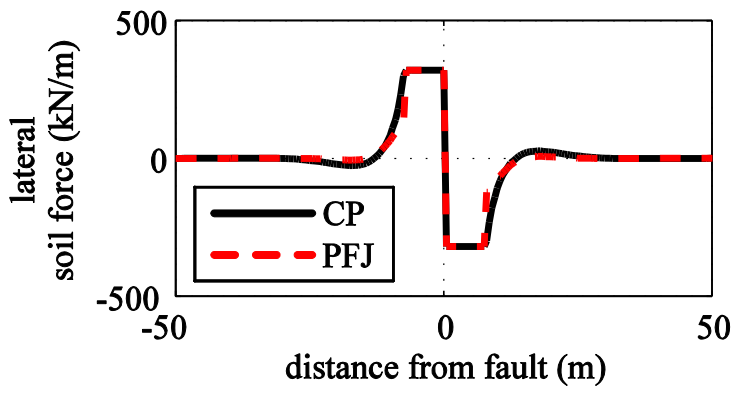

Fig. 9 Lateral soil response distribution on continuous pipeline and pipeline with flexible joints

Within the scope of the present illustrative example, strain hazard curves are derived by combining numerical results and a basic probabilistic approach, where fault displacement hazard is represented by its mean, considering epistemic uncertainties. Also, strain capacities are considered to be deterministic, rather than uncertain. The mean annual rate of exceeding a defined strain value is estimated via Eq. (9). However, in the illustrative example it applies that $\lambda_{D}=\lambda_{E}$ as the relationship between fault displacement $D$ and fault component $\Delta_{1}$ is "one to one" and thereby the proposed approximate calculation procedure of fault components is simplified.

For the pipeline under investigation, the tensile strain limit equals $2 \%$, while the compressive strain limit yields $0.39 \%$ via Eq. (8). Then, combination of numerical results with results of PFDHA leads to the production of the pipeline strain hazard curves that are illustrated in Fig. 10 and Fig. 11 for longitudinal tensile and compressive strains of $\mathrm{CP}$ and PFJ, respectively. Strain hazard curves indicate that tensile strains of CP reach the $2 \%$ limit, while the corresponding strains of PFJ are lower. On the other hand, as strike-slip offset leads to pipe bending and tension, compressive strain hazard curves indicate that there is no risk of pipe failure due to buckling. Furthermore, both tensile and compressive strains highlight the efficiency of flexible joints in concentrating strains, as developing strains on pipe steel parts are substantially decreased.

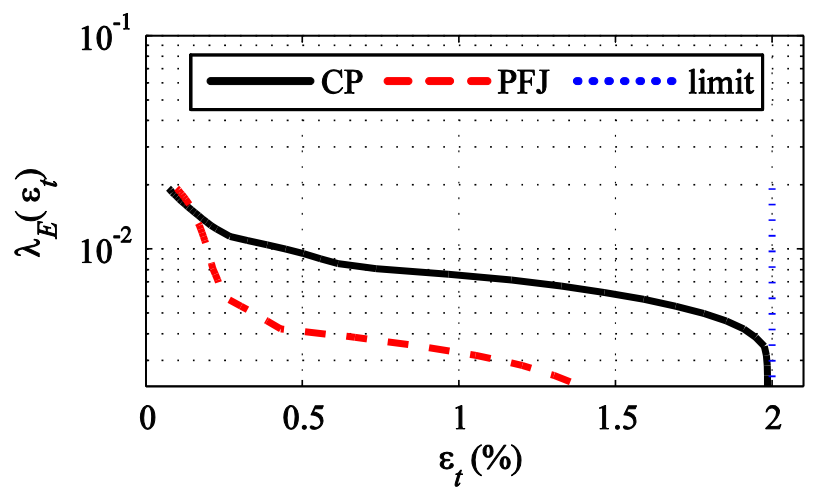

Fig. 10 Continuous pipeline and pipeline with flexible joints tensile strain hazard curves

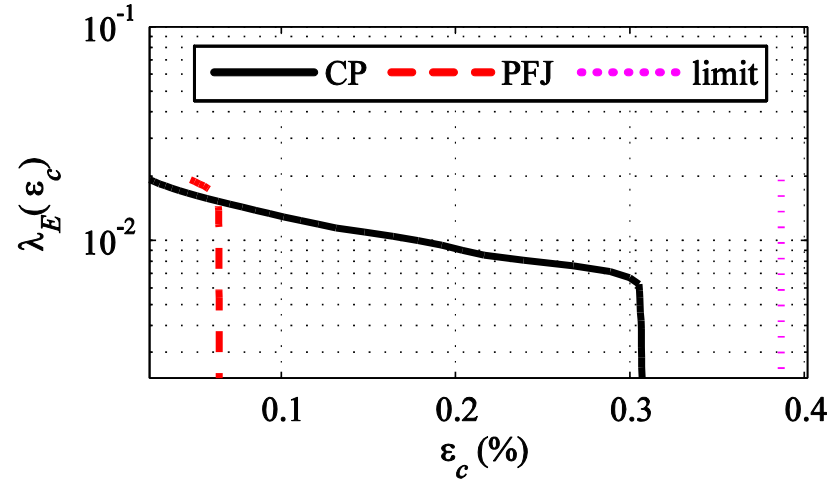

Fig. 11 Continuous pipeline and pipeline with flexible joints compressive strain hazard curves

\section{CONCLUSION}

A probabilistic evaluation methodology of innovative mitigating measures against the consequences of faulting on buried steel pipelines is presented. The first step includes the Probabilistic Fault Displacement Hazard Analysis methodology, as adjusted for pipeline fault crossing. The conceptual algorithm of the proposed methodology is presented as well, by considering also related epistemic uncertainties. The second step is the "transition" from seismological data to structural analysis through the vector intensity measure of fault displacement components. The structural analysis of the continuous pipeline indicates the appropriate locations for the integration of flexible joints as innovative mitigating measures to reduce the effects of faulting on pipelines in terms of developing bending moments and longitudinal strains. Strain hazard curves are created for both continuous pipelines and pipelines with flexible joints by associating numerical results to hazard analysis in order to compute the mean annual rate of exceeding tensile and compressive strains, which are compared to code-based strain failure criteria. Such strain hazard curves highlight the effectiveness of flexible joints as mitigating measures by reducing the potential of local buckling and tensile fracture of girth welds. The proposed two-step methodology provides engineers with a reliable estimation tool and offers a path for performance-based assessment on the efficiency of such measures, offering both seismic risk assessment and pipeline numerical analysis at low computational effort.

\section{ACKNOWLEDGMENTS}

Financial support was provided by the European Research Executive Agency via Marie Curie grant PCIG09-GA-2011293855 and by Greece and the European Social Funds through the Operational Program "Human Resources Development" of the National Strategic Framework (NSRF) 2007-2013. 


\section{REFERENCES}

[1] O'Rourke, M.J. and Liu, X., "Response of buried pipelines subject to earthquake effects, Monograph No. 3", Multidisciplinary Center for Earthquake Engineering Research, Buffalo, New York, 1999.

[2] Melissianos, V.E., Vamvatsikos, D., Gantes, C.J., "Seismic risk assessment of buried pipelines at active fault crossings", Proceedings of the Second European Conference on Earthquake Engineering and Seismology, Istanbul, Turkey, August 25-19, 2014.

[3] Youngs, R.R., Arabasz, W.J., Anderson, R.E., Ramelli, A.R., Ake, J.P., Slemmons, D.B., McCalpin, J.P., Doser, D.I., Fridrich, D.J., Swan III, F.H., Rogers, A.M., Yount, J.C., Anderson, L.W., Smith, K.D., Bruhn, R.L., Knuepfer, P.L.K., Smith, R.B., dePolo, C.M., O'Leary, D.W., Coppersmith, K.J., Pezzopane, S.K., Schartz, D.P., Whitner, J.W., Olig, S.S. and Toro, G.R., "A methodology for probabilistic fault displacement hazard analysis", Earthquake Spectra, 19(1), 191-219, 2003.

[4] Karamanos, S.A., Keil, B., Card, R.J., "Seismic design of buried steel water pipelines", Proceeding of the Pipelines 2014: From Underground to the Forefront of Innovation and Sustainability, Portland, Oregon, USA, August 3-6, 1005-1019, 2014.

[5] Gantes, C.J. and Bouckovalas, G., "Seismic verification of the high pressure natural gas pipeline KomotiniAlexandroupolis-Kipi in areas of active fault crossings", Structural Engineering International, 23(2), 204-208, 2013.

[6] Monroy-Concha, M., "Soil restraints on steel buried pipelines crossing active seismic faults", Ph.D. The University of British Columbia, Canada, 2013.

[7] Bekki, H., Kobayashi, K., Tanaka, Y. and Jain, S.K., "Dynamic behavior of buried pipe with flexible joints in liquefied ground", Journal of Japan Sewage Association, 39, 201-208, 2002.

[8] Melissianos, V.E. and Gantes, C.J., "On the efficiency of flexible joints in mitigating the consequences of seismic fault activation on buried pipelines", EEOP0052, Proceedings of the Qatar Foundation Annual Research Conference 2014, Doha, Qatar, November 18-19, 2014.

[9] Cornell, C.A., "Engineering seismic risk analysis", Bulletin of the Seismological Society of America, 58, 1583-1606, 1968.

[10]Wells, D.L. and Coppersmith, K.J., "New empirical relationships among magnitude, rupture, length, rupture width, rupture area and surface displacement", Bulletin of the Seismological Society of America, 84, 974-1002, 1994.

[11]Gutenberg, R. and Richter, C.G., "Frequency of earthquakes in California", Bulletin of the Seismological Society of America, 34, 185-188, 1944.

[12] Bazzuro, P. and Cornell, C.A., "Vector-valued probabilistic seismic hazard analysis (VPSHA)", Proceedings of the 7th U.S. National Conference on Earthquake Engineering, Boston, USA, July 21-25, 2000.
[13] Bommer, J.J. and Scherbaum, G., "The use and misuse of logic trees in probabilistic seismic hazard analysis", Earthquake Spectra, 24(4), 997-1009, 2008.

[14] Abrahamson, N.A. and Bommer, J.J., "Probability and uncertainty in seismic hazard analysis", Earthquake Spectra, 21(2), 603-607, 2005.

[15] Vazouras, P., Karamanos, S.A. and Dakoulas, P., "Finite element analysis of buried steel pipelines under strike-slip fault displacement", Soil Dynamics and Earthquake Engineering, 30, 1361-1379, 2010.

[16] Vazouras, P., Karamanos, S.A. and Dakoulas, P., "Mechanical behavior of buried steel pipes crossing active strike-slip faults", Soil Dynamics and Earthquake Engineering, 41, 164-180, 2012.

[17] Trifonov, O., "Numerical stress-strain analysis of buried steel pipelines crossing active strike-slip fault with an emphasis on fault modeling aspects", ASCE Journal of Pipeline System Engineering Practice, 6(1), 04014008, 2015.

[18] Peng, L.C. and Peng, A., "Pipe stress engineering”, ASME Press, New York, USA, 2009.

[19] American Lifelines Alliance (ALA), "Guidelines for the Design of Buried Steel Pipe - July 2001 (with addenda through February 2005", ASCE American Lifelines Alliance, July 2001.

[20] ADINA R \& D Inc., "Theory and Modeling guide Volume I: ADINA, Report ARD 08-7”, Watertown, USA, 2008. 